\title{
Arrhyyhmogenic Right Ventricular Dysplasia; Radiologic Findings Of The Left Ventricle: A Case Report And Review Of The Literature
}

\author{
KARDA, N CILEDAG, F KACMAZ, O TUFEKCIOGLU, Y SEREFLISAN
}

\begin{abstract}
Arrhythmogenic right ventricular dysplasia (ARVD) is characterized by progressive fibro fatty replacement of the right ventricular myocardium which constitutes a substrate for electrical instability and a focus of ventricular arrhythmias. Most of the patients have some degree of left ventricular involvement which also affects the right ventricle by the same disease process. From thirty percent to $50 \%$ of patients with ARVD have a family history of the disease. The most common pattern of inheritance is autosomal dominant. Clinically, ARVD usually presents with ventricular arrhythmias, and there is approving evidence that this is the underlying disease in a substantial number of sudden deaths among young, especially healthy individuals. The diagnosis of ARVD relies on the presence of structural and functional abnormalities of the right ventricle, electrophysiological abnormalities, and family history. The imaging modalities are conventional angiography, echocardiography (ECO), radionuclide angiography, ultra fast computed tomography, and magnetic resonance imaging. (MRI). We are presenting a case of ARVD with ECO, computed tomography (CT), and MRI findings.
\end{abstract}

Ind J Radiol Imag 2006 16:1:131-134

Keywords: Dysplasia, Arrhythmia, Arrhythmogenic right ventricular dysplasia, Magnetic resonance image, computed tomography, echocardiography

\section{INTRODUCTION}

ARVD is clinically characterized by ventricular arrhythmias with left bundle branch block (LBBB) which may lead to cardiac arrest and morphologically by fatty or fibro fatty infiltration of the right ventricular myocardium (1-7). Most of the patients have some degree of left ventricular involvement which also affects the right ventricle by the same disease process (4). Several reports suggest that there is a familial occurrence of ARVD of about $30 \%$ $50 \%$ (2), with mainly autosomal dominant inheritance, various penetrance, and polymorphic phenotypic expression $(1,7,8)$. Although incidence and prevalence of ARVD are unknown, ARVD is recognized as a major cause of sudden death in young adolescents, and in one series it was found that $20 \%$ of sudden deaths in the individuals younger than 35 and $22 \%$ of sudden deaths in young athletes were caused by ARVD $(1,8)$. Therefore, an early and accurate diagnosis followed by appropriate therapy for this condition is increasingly becoming important as it may prevent lethal arrhythmias. We are reporting a 28-year-old-man with ARVD. The diagnosis was achieved by CT and MR imaging, that showed fatty infiltration of right and ventricle wall and was confirmed with the echocardiography findings.

\section{CASE REPORT}

A 28 year-old-man admitted to our hospital for evaluation of long staining palpitation and recurrent syncope. His physical examination was normal other than arrhythmic cardiac pulse. T-wave inversion in the right precordial leads and ventricular premature beats with left bundle branch block morphology was present in ECG.

Holter monitor recordings revealed runs of sustained ventricular tachycardia episodes (Figure 1).

Transthoracic Echocardiography (TTE) showed right ventricular dilatation with reduced systolic function (\%3738 ejection fraction), dyskinesia and severely attenuated systolic thickening in the free wall (systolic thickness:

From the Türkiye Yüksek Ihtisas Hospital, Department of Radiology, Sihhiye Ankara TURKEY

Request for Reprints: Kemal Arda, MD, Türkiye Yüksek Ihtisas Hospital, Department of Radiology, Sihhiye Ankara TURKEY

Received 14 October 2005; Accepted 10 March 2006 
3mm) (Figure 2).

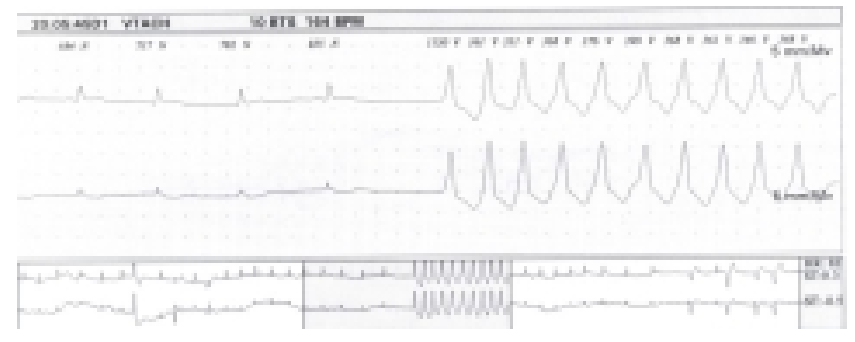

Figure 1. Holter monitorization showed episodes of sustained ventricular tachycardia in the form of LBBB (Originating from the right ventricle).

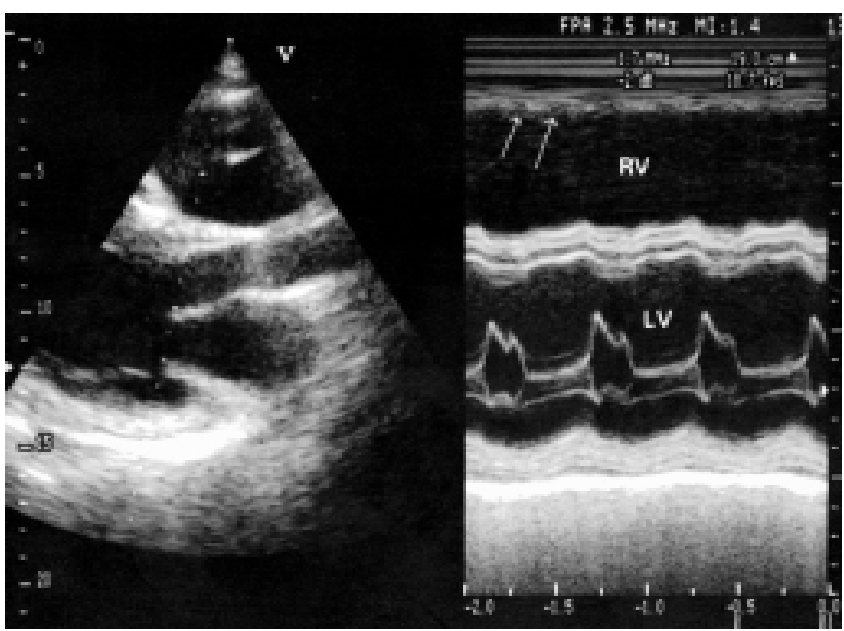

Figure 2. M-mode echocardiography shows thinning and marked reduced systolic thickening in the right ventricular wall(arrows). Note the dilatation and attenuated systolic functions of the right and left ventricles (LV, left ventricle; RV, right ventricle).

CT scans showed fatty infiltration (in minus $\mathrm{HU}$ values) in the subepicardial myocardium of the left ventricle wall and also showed that the thickness of the left ventricle wall was $2 \mathrm{~mm}$ (Figure 3).

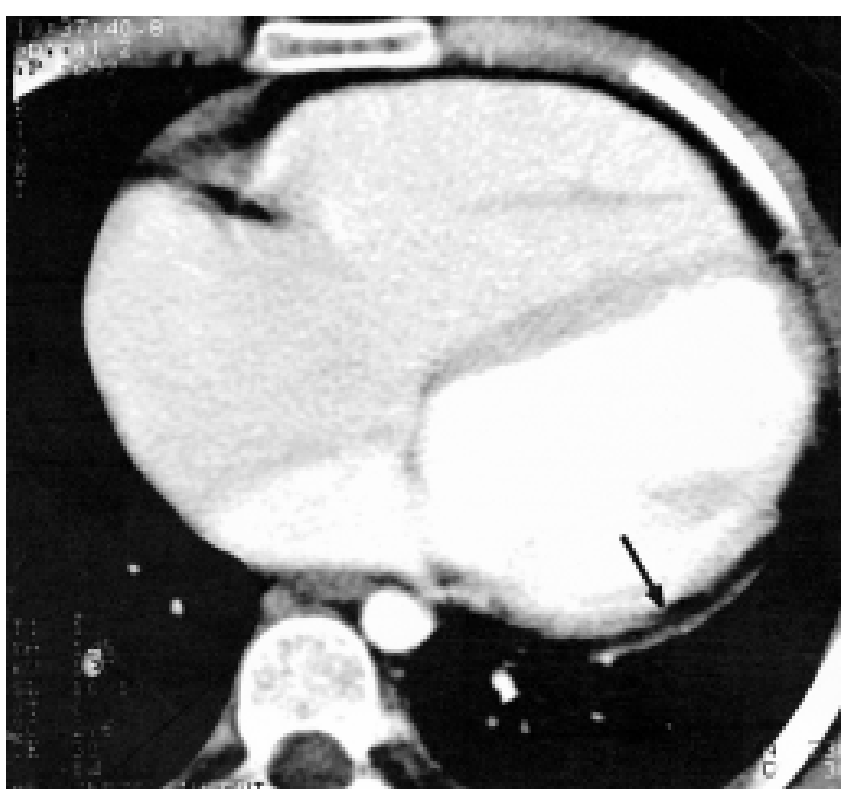

Figure 3. CT scans showed fatty infiltration in the myocardium of the left ventricle wall and also showed that the thickness of the left ventricle wall was $2 \mathrm{~mm}$.
MR imaging revealed bulging of the right ventricle free wall in end-diastole, absence of systolic thickening, fibrofatty infiltration and enlargement in the left ventricle outflow tract (Figure 4).

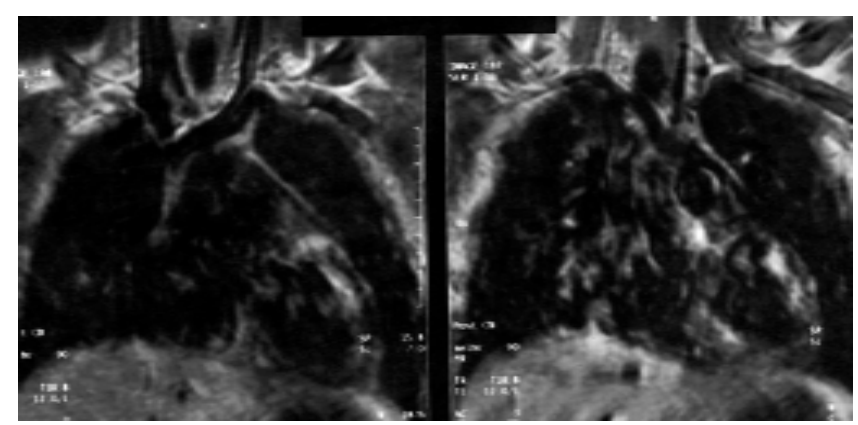

Figure 4. T1 weighted MR image shows thinning and high signal intensity of fat in the left ventricular wall.

B blocker therapy (metoprolol 100mg/day) was started and an AICD (Automated Implantable Cardioverter Defibrillator) was implanted successfully and this treatment provided good symptomatic relief.

\section{DISCUSSION}

ARVD is characterized by fibro fatty replacement of the right ventricle myocardium pathologically and by right ventricular arrhythmias clinically. In most of the ARVD patients, some degree of left ventricular involvement is seen. Biventricular involvement is usual, but isolated left ventricular involvement is a rare condition.

In our case both right and left ventricular involvement was diagnosed.

ARVD is an important cause of sudden death among young people. Pathogenesis, prevalence, and etiology are not fully known yet. The diagnosis at an early stage is essential. McKenna et al (1) reported the diagnostic criteria of ARVD, which are based on the presence of structural, histological, electrocardiograph cal findings and arrhythmic, genetic factors (Table 1). The detection of functional and structural alternations are realized with echocardiography, conventional angiography, CT, MRI, or radionuclide angiography. Two major, one major and two minor, or four minor criteria is sufficient for the diagnosis of ARVD

Table 1. Criteria for Diagnosis of Arrhythmogenic Right Ventricular Dysplasia/Cardiomyopathy(3)

I. Family history

Major criteria

Familial disease confirmed at necropsy or surgery Minor criteria 
IJRI, 16:1, February 2006

Family history of premature sudden death (at $<35$ years) causde by suspected ARVD

Family history (clinical diagnosis based on present criteria)

II. ECG depolarisation/conduction abnormalities Major criteria

Epsilon waves or localized prolongation ( $=110 \mathrm{msec}$ ) of the QRS complex in right precordial leads (V1-V3) Minor criteria

Late potentials seen on signal averaged electrocardiography

III. ECG repolarisation abnormalities

Minor criteria

Inverted T waves in right precordial leads (V2 and V3) in people older than 12 years and in the absence of right bundle branch block

IV. Arrhythmias

Minor criteria

Sustained or nonsustained left bundle branch block type ventricular tachycardia documented on the ECG, Holter monitoring or during exercise testing

Frequent ventricular extrasystoles (more than 1000/ 24 hours on Holter monitoring)

V. Global and/or regional dysfunction and structural alterations

Major criteria

Severe dilatation and reduction of right ventriclar ejection fraction with no (or only mild) left ventriclar involvement

Localized right ventriclar aneurysms (akinetic or dyskinetic areas with diastolic bulging)

Severe segmental dilatation of the right ventricle Minor criteria

Mild global right ventriclar dilatation and/or ejection fraction reduction with normal left ventricle

Mild segmental dilatation of the right ventricle

Regional hypokinesia of the right ventricle

VI. Tissue characterization of walls

Major criteria

Fibrofatty replacement of myocardium at endomyocardial biopsy

Kimura et al (5) and Kayser et al (1) reported CT and MRI findings of ARVD.

We added CT and MRI findings of our case and made a table of CT and MRI findings for the diagnosis of ARVD (Table 2) $(1,5,14)$.

\section{Table 2. CT and MRI findings of ARVC $(5,8,14)$}

CT findings

a)Fatty density in the subepicardial myocardium of the right ventricular wall,

b)To become thining of right ventricular free wall,

c) Enlarged myocardial trabeculae of anterior and inferior walls,

\section{Arrhyyhmogenic Right Ventricular Dysplasia 133}

d)Fatty tissue in the left ventricular free wall and in the ventricular septum,

e)Diastolic bulging of the free wall of the right ventricle,

g)Abnormal and reduced wall functions of the right ventricle,

h) Dilatation of right ventricle,

i)Right ventricle outflow tract > left ventricle outflow tract

MR imaging findings

I.Spin-Echo technique

a)On T1 weighted images, characteristic high signal intensity of fat in the right ventricular wall (non-invasive myocardial tissue characterisation),

b) To become diffuse thinning of the right ventricle,

c)Dilatation and aneurysm of the right ventricle,

d) Right ventricle outflow tract > left ventricle outflow tract

II.Cine MRI technique

a)Regional or global enlargement of the right ventricle,

b)Systolic and diastolic dysfunction,

c) Regional contraction abnormalities,

d)Aneurysm of right ventricle,

The standard of reference for the diagnosis of ARVD is based on histological demonstration of transmural fibro fatty replacement of the right ventricular myocardium in either surgery or autopsy. Even when the biopsy specimen is obtained from the right ventricular free wall, the sensitivity is $67 \%$ and the specificity is $92 \%$ for the diagnosis of ARVD, based on the criteria of greater than $3 \%$ fat and less than $40 \%$ fibrous tissue replacing the myocardial muscle (1). Recent studies demonstrated that significant fatty infiltration of the right ventricle occurs in more than $50 \%$ of normal hearts in elderly people. The demonstration of some fat in the right ventricular myocardium may not be specific enough to help in establishing the diagnosis of ARVD $(4,9,10)$. Therefore, in most cases, endomyocardial biopsy to confirm the diagnosis is not recommended, especially when the right ventricular wall is very thin and biopsy of this region risks perforation and cardiac tamponade. In our case due to high risk of the perforation and cardiac tamponade, endomyocardial biopsy was not performed.

The sensitivity and specificity of angiography in the diagnosis of ARVD is low. Echocardiography plays an important role in the evaluation of patients with suspected ARVD. However, it has many limitations in the assessment of right ventricular function, and there is still no generally recommended method to quantify the function of the right ventricle.

Kimura et al (5) reported characteristics of the helical CT in the diagnosis of the ARVD. These are the dilatation of the right ventricle, fatty tissue in conspicuous trabeculae of the right ventricle, especially in the anterior wall, apex, and inferior wall, and a scalloped appearance (bulging) of the right ventricular wall. The fatty tissue of the left ventricle 
and ventricular septum is frequently seen in ARVD, and fat in ventricular septum is another useful finding for the diagnosis of ARVD. Electron-beam tomography and multislice CT are superior to non-gated helical CT in assessment of abnormal right and left ventricular functions.

MR imaging allows a three-dimensional evaluation of the heart and the ventricular anatomy and volumes, and, in views of its excellent spatial resolution and unlimited field of view, MR imaging is an excellent tool to visualize the right ventricle $(1,3,10,11,13)$. MR imaging is useful for evaluating not only of fatty replacement of the right ventricular myocardium but also the global regional functional abnormalities of the right ventricle and right ventricular outflow tract. MR imaging provides the most important anatomic, functional, and morphological criteria for diagnosis of ARVD. Positive MR imaging findings should be used as important additional criteria in the clinical diagnosis of ARVD, although negative MR imaging findings do not rule out ARVD (1).

As a conclusion, CT is a simple and noninvasive technique that may give information about morphology and tissue character of the right ventricle. MRI can be performed simply and give much information about morphology, functional abnormalities and also tissue character of the right and the left ventricle wall.

\section{REFERENCES}

1. Kayser H, Wall E, Sivananthan M, Blooker T, Roos A.Diagnosis of arrhythmogenic right ventricular dysplasia.Radiographics 2002;22:639-650.

2. Linström L, Wilkenshoff $M$, Larsson $H$, Wranne B.Echocardiographic assessment of arrhythmogenic right ventricular cardiomyopathy. Heart 2001;86:31 -38.

3. Corrado D, Basso C, Thiene G.Arrhythmogenic right ventricular cardiomyopath: diagnosis, prognosis, and treatment.Heart 2000;83:588-595.

4. Pasquale CG, Heddle WF.Left sided arrhythmogenic ventricular dysplasia in siblings. Heart 2001;86:128-130.

5. Kimura F, Sakai F, Sakomura Y, Fujimura M, Ueno E, Matsuda N, Kasanuki H, Mitsuhashi N. Helical CT features of arrhythmogenic right ventricular cardiomyopathy. Radiographics 2002;22:1111-1124.

6. Markus F, Towbin J, Zareba W, Moss A, Calkins H, Brown M, Gear K. Arrhythmogenic right ventricular dysplasia/ cardiomyopathy.Circulation 2003;107:2975-2978.

7. Gilbert-Barness E. Review: Metabolic cardiomyopathy and conduction system defects in children. Annals of clinical \& laboratory science 2004;34(1):15-34.

8. Fisher NG, Gilbert TJ. Arrhythmogenic right ventricular dysplasia. An illustrated review highlighting developments in the diagnosis and management of this potentially fatal condition. Postgrad med j 2000;76:395398.

9. Basso C, Thiene G, Corrado D, Angelini A, Valente M. Arrhythmogenic right ventricular cardiomyopathy: dysplasia, dystrophy, or myocarditis? Circulation 1996;64:983-991.

10. Blake LM, Schheinmann MM, Higgins CB. MR features of arrhythmogenic right ventricular dysplasia. AJR 1994;162:809-812.

11. White RD, Trohman RG, Flamm SD, et al. Right ventricular arrhythmia in the absence of arrhythmogenic dysplasia: MR imaging of myocardial abnormalities. Radiology 1998;207:743-751.

12. Pantanowitz L. Fat infiltration in the heart. Heart 2001;85:253.

13. Diamon Y, Watanabe S, Takeda S, Hijikata Y, Komuro I. Two-layered appearance of noncompaction of the ventricular myocardium on magnetic resonance imaging. Circulation j2002;66:619-621.

14. Tandri $H$, Saranathan M, Rodriguez R, Martinez $C$, Bomma C, Nasir K, Rosen B, Lima J, Calkins Hugh, Bluemke D. Noninvasive detection of myocardial fibrosis in arrhythmogenic right ventricular cardiomyopathy using delayed-enhancement MRI. Journal of the American College of Cardiology 2005;45:98-103. 